\title{
Uma abordagem via análise envoltória de dados para o estabelecimento de melhorias em segurança baseadas na FMEA
}

\section{A data envelopment analysis approach to safety improvements based on FMEA}

\author{
Pauli Adriano de Almada Garcia ${ }^{1}$
}

\begin{abstract}
Resumo: O presente trabalho apresenta uma abordagem baseada em análise envoltória de dados (Data Envelopment Analysis - DEA) para estabelecer direcionamentos de melhoria para os modos de falhas identificados na análise dos modos e efeitos de falha (FMEA). A abordagem tradicional da FMEA baseia-se num número de priorização de riscos que vem sendo alvo de inúmeras críticas embasadas em diversos artigos científicos. No presente trabalho, o número de priorização de risco é baseado em DEA. Por meio desse número de priorização de risco DEA, considerando-se o conceito de fronteira de eficiência, serão identificadas diretrizes de melhoria para os modos de falha com base nos índices de risco estabelecidos pela equipe executora da FMEA. Por meio dos resultados alcançados nas duas aplicações práticas, pode-se demonstrar a eficácia da abordagem proposta para tratar essa classe de problemas.

Palavras-chave: Análise de falha. FMEA. Análise envoltória de dados. Número de priorização de riscos. Análise de risco.
\end{abstract}

\begin{abstract}
The present study presents a data envelopment analysis (DEA) based approach to establish guidelines for the improvement of the failure modes identified by a failure mode and effect analysis (FMEA). The traditional FMEA approach is based on risk priority number $(R P N)$, which has been target of scientific criticism. In the present study, the RPN is based on DEA and considers the concept of efficiency frontier. Based on this concept and on the risk indexes, some improvement directives for the failure modes can be established. The obtained results concerning the two practical applications demonstrate the effectiveness of the proposed approach in dealing with this kind of problem.
\end{abstract}

Keywords: Failure analysis. FMEA. Data envelopment analysis. Risk priority number. Risk analysis.

\section{Introdução}

Análise de risco é uma atividade que é abundantemente realizada por engenheiros de confiabilidade e analistas de risco de qualquer indústria. Os resultados de uma Análise Probabilística de Segurança (APS) fornecem informações para que uma decisão seja tomada com respeito a políticas de manutenção ou sobre cuidados a serem tomados num determinado ponto crítico do sistema sob análise (FULLWOOD, 2000). Uma análise dos modos e efeitos de falha (Failure Mode and Effect Analysis - FMEA), numa análise probabilística de segurança (APS), tem por finalidade identificar e prover informações semiquantitativas com respeito às diferentes formas e modos de como o sistema sob análise pode vir a falhar, além de constituir os fatores de entrada para a modelagem do sistema, IAEA (INTERNATIONAL..., 1992).

Os dados levantados pela realização de uma FMEA devem ser todos considerados para uma tomada de decisão envolvendo risco. Os dados que devem influenciar o tomador de decisões estão relacionados com a probabilidade de ocorrência de um determinado modo de falha, com a gravidade dos efeitos da ocorrência deste modo de falha e com a capacidade de identificação de uma causa potencial da falha, de modo a prevenir a ocorrência do evento (BOWLES, 1998; BOWLES; BONNELL, 1998).

Até o presente momento, diferentes técnicas têm sido utilizadas para minimizar as interpretações errôneas ocasionadas pelo método tradicional de priorização de risco, que é o denominado Número de Priorização de Riscos (Risk Priority Number - RPN) (BOWLES; BONNEL, 1998; BOWLES, 2003).

O RPN tradicional consiste do produto de três atributos associados aos modos de falha levantados durante a realização de uma FMEA. Estes atributos são representados por índices que estão relacionados com a probabilidade de ocorrência $(\mathrm{O})$, a gravidade

\footnotetext{
${ }^{1}$ Departamento de Administração, Polo Universitário de Volta Redonda - PUVR, Universidade Federal Fluminense - UFF, CEP 27213-415, Volta Redonda, RJ, Brasil, e-mail: pauliadriano@gmail.com
} 
(G) dos efeitos associados à ocorrência e com o potencial de detecção (D) do modo de falha, conforme previamente mencionado. Estes índices variam numa escala numérica inteira de 1 a 10, ou, em alguns casos, podem estar numa escala de 1 a 5 , em que quanto maior o índice, pior a situação.

Mesmo para o caso do índice de detecção, quanto maior o valor do índice menor é o potencial de resposta do sistema. Isso se dá, pois, na abordagem tradicional, deseja-se estabelecer uma ordem de prioridade entre os modos de falha por meio do RPN. Desse modo, mesmo que intuitivamente, seja a percepção, para o caso do índice de detecção, de quanto maior, melhor a situação, essa consideração levaria a confusões na interpretação dos diferentes RPN. Sendo assim, as diferentes normas que tratam do assunto, Society for Automotive Engineers (SAE) (SOCIETY..., 2009), QS9000 (INSTITUTO..., 1997) etc. trazem tabelas com as definições conforme previamente mencionado, ou seja, quanto maior o índice, pior a situação.

O problema associado ao RPN foi primeiramente levantado por Bowles e Peláez (1995, p. 204):

[...] no cálculo do RPN, um modo de falha que possui um índice de gravidade muito elevado, uma baixa probabilidade de ocorrência e um elevado poder de detecção $(\mathrm{G}=9, \mathrm{O}=3$ e $\mathrm{D}=2$ que conduz a um $\mathrm{RPN}=54$ ) pode ser considerado menos crítico que outro modo de falha que possua estes mesmos índices em níveis medianos $(\mathrm{G}=4, \mathrm{O}=5$ e $\mathrm{D}=6$, que conduzem a um RPN =120)

Contudo, em termos de ações a serem tomadas, o primeiro modo de falha, considerado por Bowles (1998), será, de forma intuitiva, priorizado para que ações corretivas sejam tomadas no intuito de se reduzir os efeitos de danos potenciais associados à sua ocorrência (ou são inseridas barreiras de segurança mais efetivas, planos de emergência para mitigar os efeitos etc.).

Um segundo ponto crítico associado à priorização dos modos de falha baseada no RPN é o fato de ter-se repetidos valores de priorização, ou seja, RPN, para modos de falha com características totalmente diferentes. Segundo Bowles (2003), dos 1000 RPN que deveriam ser produzidos pelo produto GxOxD, somente 120 deles são únicos. De acordo com a Tabela 1 , tem-se, por exemplo, que o RPN $=64$ pode ser gerado de 10 maneiras distintas.

Um terceiro ponto crítico associado ao RPN é que nenhum número maior do que 10 tendo um primo como fator poderá ser formado pelo produto dos três parâmetros. Assim, os números 11, 22, 33, 990 etc. são valores de RPN que não podem ser gerados pelo produto dos índices de ocorrência, gravidade e detecção. Pelo mesmo motivo, os múltiplos de 13, 17, 19 etc. serão excluídos (BOWLES, 2003).
Tabela 1. Combinações de gravidade, ocorrência e detecção que geram $\mathrm{RPN}=64$.

\begin{tabular}{cccc}
\hline Gravidade & Ocorrência & Detecção & RPN \\
\hline 1 & 8 & 8 & 64 \\
2 & 4 & 8 & 64 \\
2 & 8 & 4 & 64 \\
4 & 2 & 8 & 64 \\
4 & 4 & 4 & 64 \\
4 & 8 & 2 & 64 \\
8 & 1 & 8 & 64 \\
8 & 2 & 4 & 64 \\
8 & 4 & 2 & 64 \\
8 & 8 & 1 & 64 \\
\hline
\end{tabular}

Fonte: Bowles (2003).

Com o intuito de sanar as deficiências supracitadas, diferentes autores propuseram abordagens diferenciadas para o estabelecimento de um número de priorização de risco. Bevilacqua, Braglia e Gabbrielli (2000) definiram o RPN como uma soma ponderada de seis parâmetros (segurança, importância do equipamento para o processo, custo de manutenção, frequência de falha, tempo de reparo, e condições operacionais) multiplicada pelo sétimo fator (dificuldade de acesso ao equipamento), em que a importância relativa dos seis atributos é estimada utilizando-se uma comparação pareada.

Braglia, Frosolini e Montanary (2000) propôs uma abordagem multiatributo baseada em análise hierárquica de processo (AHP - Analitic Hierarchy Process) e denominou de multi-attribute failure mode analysis (MAFMA). Nessa abordagem, os fatores de risco (ocorrência - $\mathrm{O}$, gravidade $-\mathrm{G}$, detecção - D e custos esperados) são considerados como atributos de decisão, as causas de falha são as alternativas e a seleção da causa de falha é o objetivo, os quais juntamente com os atributos e alternativas compõem uma estrutura hierárquica de três níveis. Matrizes de comparações pareadas são utilizadas para estimar os pesos dos atributos e as prioridades das causas com relação ao custo esperado.

Sankar e Prabhu (2001) apresentaram uma abordagem diferenciada para a priorização dos modos de falha, baseando-se numa ordenação de 1 a 1000 para representar o incremento no risco em 1000 combinações possíveis para os índices de ocorrência, gravidade e detecção. Essas 1000 possíveis combinações foram tabuladas por um especialista em ordem crescente e interpretadas por meio de regras do tipo "se-então". Quanto maior a ordem, maior a prioridade do modo de falha.

Garcia (2001), Garcia, Neves e Luz (2001) e Garcia e Neves (2001) apresentaram abordagens baseadas em análise envoltória de dados para priorizar os modos de falha por meio de uma medida de ineficiência 
relativa. Nas abordagens apresentadas, os autores propuseram o modelo de agregação parcial com gravidade fixa (APGF).

Chang, Liu e Wei (2001) utilizam a análise relacional grey para o estabelecimento do grau de relacionamento grey entre os modos de falha identificados e um modo de falha padrão, que representa o estado meta.

Inúmeros outros trabalhos foram publicados considerando-se as incertezas associadas às informações provenientes dos especialistas. Esses trabalhos, de modo geral, baseiam-se de alguma forma na teoria dos conjuntos fuzzy. Dentre esses trabalhos pode-se destacar: (i) Bowles e Peláez (1995), que apresentaram uma abordagem fuzzy que se baseia em variáveis linguísticas para representar os índices de ocorrência, gravidade e detecção, além de estabelecer uma variável linguística para caracterizar o risco. O relacionamento entre as variáveis $\mathrm{O}, \mathrm{G}$ e $\mathrm{D}$, inferindo no risco, se dá por meio de regras do tipo "se-então", extraídas por meio de opinião de especialistas. Outros trabalhos com as mesmas características de Bowles e Peláez (1995) foram desenvolvidos como, por exemplo, Guimarães e Lapa (2004a, 2007), Braglia, Frosolini e Montanary (2003), entre outros. (ii) Cheng, Wei e Lee (1999) combinam os conceitos associados aos conjuntos fuzzy com a análise relacional grey. Os índices de $\mathrm{O}$, G e D são abordados via variáveis linguísticas e suas respectivas funções de pertinência. (iii) Garcia, Schirru e Frutuoso e Melo (2005) e Garcia, Frutuoso e Melo e Schirru (2009) propuseram uma abordagem fuzzy DEA para a priorização dos modos de falha, baseando-se em variáveis linguísticas para $\mathrm{O}, \mathrm{G}$ e D. O diferencial da abordagem proposta está no fato de não ser necessário o estabelecimento de um conjunto fuzzy de saída, necessário para o sistemas baseado em regras do tipo "se-então", além de também não ser necessária a observação das informações crisp para servirem de entrada no sistema. (iv) Yang, Bonsall e Wang (2008) propuseram uma abordagem para a FMEA utilizando raciocínio Bayesiano baseado em regras fuzzy (fuzzy rule-based Bayesian reasoning - FuRBaR) para a priorização dos modos de falha. A técnica foi especificamente desenvolvida para lidar com os inconvenientes relacionados às regras especialistas adotadas na inferência fuzzy.

Outras abordagens também vêm sendo propostas como, por exemplo, Chin et al. (2009a) que propuseram uma FMEA utilizando raciocínio de grupo baseado em evidências para levantar as diversidades das opiniões dos membros da equipe de análise e priorizar os modos de falha considerando diferentes tipos de incertezas. Chin et al. (2009b) também propuseram uma abordagem diferenciada da proposta por Garcia (2001), Garcia, Neves e Luz (2001) e Garcia e Neves (2001). Na abordagem proposta pelos primeiros, é utilizada uma média geométrica entre as abordagens DEA otimista e pessimista para se estabelecer um índice de risco total. Nesse mesmo artigo, os autores também propõem uma abordagem intervalar DEA.

No presente trabalho, o que se propõem é a utilização do modelo DEA tradicional, ou seja, modelo DEA CCR (CHARNES; COOPER; RHODES, 1978) sob a ótica dos outputs. Nessa abordagem, a fronteira de eficiência será formada pelos modos de falha menos críticos. Com isso, o que se pretende é identificar os pontos de melhoria para os modos de falha mais críticos, envoltos pela fronteira, além de buscar identificar de que forma seus respectivos índices $\mathrm{O}, \mathrm{G}$ e $\mathrm{D}$ precisam ser melhorados para que alcancem a fronteira e deixem de ser considerados os mais críticos.

\section{Modelos tradicionais de dea para análise de desempenho}

A análise envoltória de dados tem seus primórdios em 1957 com Farrell (FARRELL, 1957), nela foi apresentada uma medida de eficiência técnica para o caso em que um único insumo (input) era utilizado para gerar um único produto (output). Em 1978, apresentou-se uma extensão da abordagem de Farrell para que diferentes inputs e outputs possam ser considerados (CHARNES; COOPER; RHODES, 1978). O modelo desenvolvido é baseado em programação linear na qual se deseja maximizar os outputs, dado um nível de input, ou minimizar os inputs, dado um nível de output. A modelagem matemática orientada para os outputs desenvolvida pode ser vista na formulação (1), Charnes et al. (1994), Cooper, Seiford e Tone (2007).

$$
\begin{aligned}
& \operatorname{Min} \varphi_{0}=\sum_{i=1}^{m} u_{i} x_{i 0} \\
& \text { subject to } \\
& \sum_{i=1}^{m} u_{i} x_{i 0}-\sum_{r=1}^{s} v_{r} y_{r j} \geq 0, j=0, \ldots, n \\
& \sum_{r=1}^{s} v_{r} y_{r 0}=1 \\
& u_{i}, v_{r} \geq \varepsilon, r=1, . ., s, i=1, \ldots, m
\end{aligned}
$$

Em que $x_{i j}$ e $y_{r j}$ são respectivamente os inputs e os outputs, r é o número de atributos considerados como outputs, i é o número de atributos considerados como inputs, e j é o número de unidades tomadoras de decisão (DMU - Decision-making Units), $\varepsilon$ é uma grandeza não arquimediana para garantir que as DMUs a compor a fronteira sejam somente as que forem fortemente eficientes (LINS; ANGULO MEZA, 2001). O subscrito 0 representa a DMU sendo avaliada, $v_{r}$ é peso atribuído ou output $y_{r}, u_{i}$ é o peso atribuído ao input $x_{i}$. Caso exista um conjunto de pesos que 
faça com que $\varphi_{0}=1$, então a $D M U_{0}$ é dita eficiente, caso contrário, ela será dita ineficiente.

Um exemplo para visualizar o que se pretende é apresentado na Figura 1.

No exemplo da Figura 1, são considerados dois inputs e um output, e a fronteira de eficiência é estabelecida com base nos dados de cada DMU considerada na análise. Note-se que o ponto D está envolto pela fronteira, caracterizando com isso que esta DMU não tem uma combinação eficiente de inputs e outputs. Observe que o ponto D' é a projeção radial do ponto D sobre a fronteira. Essa projeção é utilizada para se determinar o quanto os atributos da DMU D precisam melhorar para que ela mesma alcance a fronteira. É exatamente esse raciocínio que será utilizado para o estabelecimento de melhoria para os modos de falha.

Na ótica input, o modelo DEA CCR tem a estrutura apresentada pela formulação (2).

$$
\begin{aligned}
& \operatorname{Max} \theta_{0}=\sum_{r=1}^{s} v_{r} y_{r 0} \\
& \text { subject to } \\
& \sum_{r=1}^{s} v_{r} y_{r j}-\sum_{i=1}^{m} u_{i} x_{i j} \geq 0, j=0, \ldots, n \\
& \sum_{i=1}^{m} u_{i} x_{i 0}=1 \\
& u_{i}, v_{r} \geq \varepsilon, r=1, \ldots, s, i=1, \ldots, m
\end{aligned}
$$

Na formulação (2), o que se busca é uma combinação de pesos que maximize os outputs fixando-se os inputs, contrariamente ao preconizado na formulação (1), em que se minimizam os inputs fixando-se os outputs.

Comumente, o uso de DEA para priorizar os modos de falha baseia-se no retorno constante de escala. Isso se dá pela interpretação do problema. Como os modos de falha estão associados a um mesmo sistema, a priori, não há justificativa para se considerar qualquer tipo de variação de escala (GARCIA, 2001; GARCIA; NEVES; LUZ, 2001; GARCIA; NEVES, 2001; GARCIA; LEAL JUNIOR; OLIVEIRA, 2012). Portanto, os modelos até então considerados são com retorno constante de escala, CRS.

Observe na Figura 2 que a medida de eficiência da DMU D é dada pela razão entre os segmentos OD e OD'. Note que, seguindo esse raciocínio, a razão entre OA e OA', em que A' coincide com A, é a unidade. Em outras palavras, a DMU A é $100 \%$ eficiente.

Diferentes modelos DEA estão disponíveis e vêm sendo desenvolvidos para as análises de eficiência e para o auxílio à tomada de decisão multicritério. Para mais detalhes sobre os modelos DEA, vide Lins e Angulo Meza (2001). Na próxima seção, apresenta-se a utilização dos modelos DEA acima, dando especial

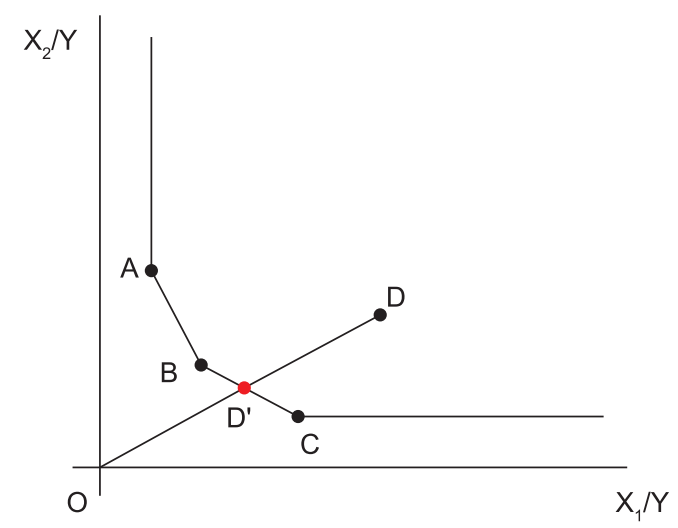

Figura 1. Fronteira de eficiência para a ótica output.

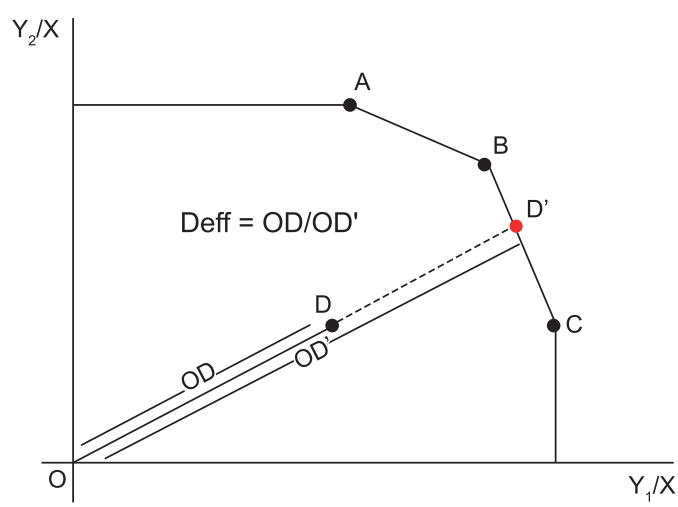

Figura 2. Fronteira de eficiência para a ótica input.

atenção à formulação 1 , ou seja, ótica output, que minimiza os inputs mantendo fixos os outputs.

\section{Aplicação de modelos dea para priorização de modos de falha}

Para aplicação dos modelos DEA como técnica de priorização dos modos de falha deve-se: (i) considerar as DMUs como sendo os modos de falha levantados durante a realização da FMEA; (ii) considerar os índices de ocorrência, gravidade e detecção como sendo atributos para a priorização dos modos de falha. Esses atributos podem ser considerados tanto como de entrada quanto de saída. No caso de se considerar os atributos como sendo fatores de saída, deve-se utilizar o modelo DEA com ótica input, ou seja, maximizando os fatores de saída e mantendo fixo os fatores de entrada, conforme formulação (2). Nesse caso, o resultado considera como integrantes da fronteira os modos de falha mais críticos, sendo esses os que devem receber maior prioridade. Para o caso em que os índices são considerados como sendo os fatores de entrada, deve-se utilizar o modelo DEA com ótica output, ou seja, minimização dos fatores de entrada mantendo-se fixos os fatores de saída, conforme formulação (1). Nesse caso, os modos 
de falha que estiverem na fronteira deverão ser considerados como sendo os menos críticos, ou seja, serão os benchmarks para os modos de falha mais críticos. Essa abordagem é a que será apresentada na próxima seção por meio de um exemplo de aplicação. (iii) deve-se considerar um atributo comum e igual para todas as DMUs. No caso de se estar trabalhando com a formulação (1), esse atributo será considerado como fator de saída. Caso contrário, ou seja, se estiver sendo considerada a formulação (2), esse fator deve ser considerado como fator de entrada. Esse fator comum pode ser interpretado como sendo o contexto operacional do sistema sob análise e é comum para todos os modos de falha.

Por exemplo, considere que tenham sido levantados os modos de falha conforme a Tabela 2.

Note que, sem perda de generalidade, foi considerado como input um valor igual a 1 para todos os modos de falha (GARCIA, 2001). O uso de input unitário no presente caso dá-se pelo fato de se estar considerando os modelos DEA como sendo de apoio multicritério à decisão. Sendo assim, os critérios a serem considerados na análise são os três já mencionados. A interpretação, no presente caso, de um input, ou output unitário, conforme será discutido adiante, está relacionada ao contexto operacional. Como está sendo avaliado um único sistema, considera-se que o sistema, e suas características intrínsecas e comuns, ocasionam a interpretação dos especialistas que compõem a equipe de FMEA. Essas interpretações associam a cada modo de falha os índices que são utilizados para sua priorização. Lovell e Pastor (1999), utilizando o modelo de envelope, mostra que não faz sentido o uso dos modelos DEA tradicionais sem input com orientação para output, bem como sem output com orientação para input. Nessas circunstâncias, a não consideração de inputs, ou output, unitários faz com que a DEA seja incapaz de diferenciar unidades eficientes das ineficientes. Soares de Mello (2006) apresenta também discussões sobre o uso de modelos dos multiplicadores com input ou output unitários. Gomes Júnior e Soares de Mello (2007) também apresentam uma discussão sobre o tema. Concluem que não há qualquer contradição em se considerar input ou output unitário. Entretanto, perde o sentido falar em ótica. No presente caso, a indicação da ótica faz referência, no caso dos multiplicadores, ao fato de se estar maximizando a soma ponderada dos outputs, para o caso da ótica input, e minimizando a soma ponderada dos inputs, para o caso da ótica output.

Nesse primeiro exemplo, será aplicado o modelo DEA CCR com ótica para os inputs, ou seja, será aplicada a formulação (2). Aplicando o referido modelo chega-se ao resultado da Tabela 3.

Nessa aplicação, os modos de falha com índice DEA igual a 1 são os que devem ser considerados mais críticos. Quanto mais afastado de 1 estiver o índice DEA, menos crítico deve ser considerado o modo de falha.

Considerando a formulação (1), deve-se então considerar os índices levantados durante a FMEA como inputs e, como output, será considerado um valor unitário e igual para todas as DMUs (os modos de falha).

Nesse caso, o output foi considerado igual para todos os modos de falha e os atributos levantados durante a FMEA foram considerados como input. No exemplo, os modos de falha com índice DEA igual a 1 devem ser considerados os menos críticos,

Tabela 2. Exemplo de modos de falha e respectivos atributos.

\begin{tabular}{ccccc}
\hline Modos de falha & Input $\mathbf{1}$ & Gravidade & Ocorrência & Detecção \\
\hline MF1 & 1 & 9 & 3 & 2 \\
MF2 & 1 & 3 & 5 & 5 \\
MF3 & 1 & 4 & 6 & 5 \\
MF4 & 1 & 5 & 4 & 6 \\
MF5 & 1 & 4 & 3 & 3 \\
MF6 & 1 & 2 & 5 & 4 \\
\hline
\end{tabular}

Tabela 3. Índices de risco estabelecidos pela formulação (2).

\begin{tabular}{cccccc}
\hline Modos de falha & Input 1 & Gravidade & Ocorrência & Detecção & DEA \\
\hline MF1 & 1 & 9 & 3 & 2 & 1 \\
MF2 & 1 & 3 & 5 & 5 & 0,94 \\
MF3 & 1 & 4 & 6 & 5 & 1 \\
MF4 & 1 & 5 & 4 & 6 & 1 \\
MF5 & 1 & 4 & 3 & 3 & 0,68 \\
MF6 & 1 & 2 & 5 & 4 & 0,83 \\
\hline
\end{tabular}


enquanto que os demais são os mais críticos. Quanto mais afastado de 1 mais crítico deve ser considerado o modo de falha. Para essa abordagem, o interessante é verificar como os modos de falha mais críticos, ou seja, os modos de falha envoltos pela fronteira, podem ser trabalhados para que deixem de ser relativamente críticos.

Outro ponto a ser observado é que, como era de se esperar, os resultados foram diferentes daqueles obtidos por meio da formulação (2). No primeiro caso, os resultados apresentados na Tabela 3 mostram que os modos de falha mais críticos foram os MFs 1, 3 e 4, enquanto que, no caso da formulação (1), conforme apresentado na Tabela 4, os modos de falha mais críticos foram os MFs 2, 3 e 4. Deve-se lembrar que, no primeiro caso, os índices $\mathrm{O}, \mathrm{G}$ e $\mathrm{D}$ foram considerados como outputs e, no segundo caso, como inputs, o que justifica essa diferença nos resultados. O enfoque da análise deve ser estabelecido como premissa inicial da análise de risco, tendo em vista que essas diferenças são passíveis de ocorrer. Cabe destacar que não há certo nem errado, e que uma FMEA é uma abordagem que é inicial, ou seja, de identificação de pontos fracos a serem trabalhados por meio de modelos mais voltados para abordagens probabilísticas (ELECTRIC..., 1996; INTERNATIONAL..., 1992, 2001, 2002).

Mantendo-nos nesse exemplo, realizando a análise para identificar os pontos de melhoria para cada modo de falha, chega-se aos resultados da Tabela 5.

Note, na Tabela 5, que os modos de falha considerados relativamente não críticos, ou seja, os modos de falha com índice DEA 1, são seus próprios alvos, conforme interpretação apresentada na Figura 2. Essa interpretação é válida, pois os modelos aqui apresentados são não arquimedianos.
No entanto, os modos de falha com índice DEA diferentes de 1 são os que estão envoltos pela fronteira e, consequentemente, são os considerados críticos. Assim, para que alcancem a fronteira e deixem de ser relativamente críticos, é preciso que algumas reduções em seus índices sejam feitas. Por exemplo, no caso do modo de falha MF3, que é o mais crítico, pois é o que tem índice DEA mais distante de 1 , ele deve ter seus índices de gravidade, ocorrência e detecção reduzidos de $28,6 \%, 31 \%$ e $28,6 \%$, respectivamente, para que ele deixe de ser relativamente crítico.

Por sua vez, o modo de falha MF4, que é o segundo mais crítico, deve ter seus respectivos índices reduzidos de $22,5 \%, 21,8 \%$ e $48,9 \%$. Enquanto que o modo de falha MF2 deve ter seus índices reduzidos de 11,9\%, $12,9 \%$ e $26,4 \%$, respectivamente.

Todos os resultados alcançados na presente seção e os resultados a serem apresentados nos exemplos de aplicação foram obtidos por meio do software AMPL com o solver CPLEX 12.2 da IBM.

\section{Exemplos de aplicação}

Nessa seção, o modelo DEA dado pela formulação 1 será aplicado em dois casos. O primeiro no sistema auxiliar de água de alimentação (AFWS - Auxiliary Feed Water System) de uma típica usina nuclear, e o segundo, no sistema de controle químico e volumétrico (CVCS - Chemical and Volumetric Control System) também de uma típica usina nuclear do tipo água leve pressurizada.

Primeiramente o modelo da formulação (1) será aplicado numa simplificação do AFWS. Segundo Guimarães e Lapa (2004a), o AFWS simplificado apresenta 12 modos de falha cujos índices de ocorrência $(\mathrm{O})$, gravidade $(\mathrm{G})$ e detecção $(\mathrm{D})$ estão dispostos na Tabela 6.

Tabela 4. Índices de risco estabelecidos pela formulação (1).

\begin{tabular}{cccccc}
\hline Modos de falha & Output $\mathbf{1}$ & Gravidade & Ocorrência & Detecção & DEA \\
\hline MF1 & 1 & 9 & 3 & 2 & 1 \\
MF2 & 1 & 3 & 5 & 5 & 0,875 \\
MF3 & 1 & 4 & 6 & 5 & 0,714 \\
MF4 & 1 & 5 & 4 & 6 & 0,778 \\
MF5 & 1 & 4 & 3 & 3 & 1 \\
MF6 & 1 & 2 & 5 & 4 & 1 \\
\hline
\end{tabular}

Tabela 5. Alvos e percentuais de melhoria para os modos de falha.

\begin{tabular}{cccccccc}
\hline Modos de falha & Índice DEA & Alvo G & Alvo O & Alvo D & Percentual G & Percentual O & Percentual D \\
\hline MF1 & 100 & 9 & 3 & 2 & 0 & 0 & 0 \\
MF2 & 85,71 & 2,64 & 4,36 & 3,68 & $-11,9$ & $-12,9$ & $-26,4$ \\
MF3 & 71,43 & 2,86 & 4,14 & 3,57 & $-28,6$ & -31 & $-28,6$ \\
MF4 & 74,15 & 3,87 & 3,13 & 3,06 & $-22,5$ & $-21,8$ & $-48,9$ \\
MF5 & 100 & 4 & 3 & 3 & 0 & 0 & 0 \\
MF6 & 100 & 2 & 5 & 4 & 0 & 0 & 0 \\
\hline
\end{tabular}


Conforme apresentado na seção anterior, no presente caso, será discutida a aplicação da formulação (1), considerando os índices da Tabela 6 como inputs e, como output, será considerado um valor igual para todos os modos de falha (DMUs).

Note-se que na Tabela 6 há seis combinações distintas entre os índices, ou seja, é como se fossem consideradas somente seis modos de falha com características distintas em relação aos índices de risco. Com base nisso, tem-se o resultado apresentado na Tabela 7.

De acordo com a formulação (1), os modos de falha considerados relativamente críticos são MF2, MF3, MF7, MF8, MF11 e MF12. Para que esses modos de falha deixem de ser considerados críticos, é preciso que ocorram melhorias em seus índices, ou seja, é preciso que os seus respectivos índices obtidos na FMEA sejam reduzidos conforme as informações da Tabela 8.

Com base nos dados da Tabela 8, os modos de falha MF2 e MF3 precisam reduzir, respectivamente, os índices de gravidade e detecção em $40 \%$ e $50 \%$.

Tabela 6. Índices de risco para o AFWS (GUIMARÃES; LAPA, 2004a).

\begin{tabular}{cccc}
\hline Modos de falha & O & G & D \\
\hline MF1 & 3 & 2 & 8 \\
MF2 & 3 & 10 & 4 \\
MF3 & 3 & 10 & 4 \\
MF4 & 3 & 6 & 2 \\
MF5 & 3 & 6 & 2 \\
MF6 & 3 & 6 & 2 \\
MF7 & 4 & 8 & 2 \\
MF8 & 4 & 8 & 2 \\
MF9 & 6 & 4 & 4 \\
MF10 & 6 & 4 & 4 \\
MF11 & 6 & 6 & 4 \\
MF12 & 6 & 6 & 4 \\
\hline
\end{tabular}

Tabela 7. Índice de risco DEA para os modos de falha do AFWS.

\begin{tabular}{cc}
\hline Modos de falha & Índice DEA \\
\hline MF1 & 100 \\
MF2 & 99,83 \\
MF3 & 99,83 \\
MF4 & 100 \\
MF5 & 100 \\
MF6 & 100 \\
MF7 & 99,9 \\
MF8 & 99,9 \\
MF9 & 100 \\
MF10 & 100 \\
MF11 & 80 \\
MF12 & 80 \\
\hline
\end{tabular}

Os modos de falha MF7 e MF8 precisam reduzir, respectivamente, os índices de ocorrência e gravidade em 25\% em ambos, e por fim os modos de falha MF11 e MF12 precisam reduzir todos os índices em $20 \%$.

Note-se que, por se estar tratando de um sistema simplificado, com um número reduzido de modos de falha, o poder discriminatório da DEA não é dos melhores. Entretanto, cabe destacar que é comum em DEA ter DMUs com eficiências e, consequentemente, ineficiências, empatadas. Em situações como essas, devem ser levados em consideração outros critérios de desempate. Especificamente na área nuclear existem especificações técnicas relacionadas a determinados modos de falha que serão determinantes em situações de desempate dos modos de falha. Ainda na área nuclear, poderá haver situações em que será necessário o investimento em mais do que um modo de falha. Caso os recursos não estejam disponíveis para tal, a planta deverá ser desligada por questões de segurança. Cabe observar no presente caso que as DMUs com eficiências diferentes da unidade empatadas têm os níveis de seus inputs iguais. Obviamente que, nesses casos, é natural que os valores das eficiências DEA sejam iguais.

Para verificar, de forma mais contundente, o potencial da aplicação da formulação (1) para análise de sistemas, a seguir, será realizada a mesma análise para o CVCS. Segundo Guimarães e Lapa (2004b), foram identificados 82 modos de falha para o CVCS. Os índices atribuídos a esses modos de falha podem ser vistos na Tabela 9. Note que ainda há modos de falha com índices de níveis iguais. Para esses será natural que os resultados sejam iguais.

Aplicando a formulação (1) para analisar os modos de falha do CVCS, chega-se ao resultado da Tabela 10.

Observe que, para o presente caso, o poder discriminatório da DEA foi bem melhor do que no caso do AFWS. Isso se deu por estar sendo considerado um número bem maior de DMUs (modos de falha) em relação ao número de critérios sendo considerado na análise. Deve-se observar também que há modos de falha com os mesmos níveis em seus critérios, o que conduzirá, naturalmente, a um mesmo índice de risco DEA, ou seja, a uma mesma priorização.

\section{Discussões e conclusões}

O presente trabalho apresentou a aplicação dos modelos tradicionais DEA em análise de modos de falha, baseando-se nos índices de risco estabelecidos durante a realização de uma FMEA. Especificamente a aplicação do modelo DEA CCR com orientação para os outputs, ou seja, manutenção dos atuais valores de saída e minimização dos insumos recebeu maior destaque, por possibilitar identificar o quanto os índices dos modos de falha considerados relativamente mais críticos precisam ser melhorados para que deixem de sê-lo. 
$\mathrm{Na}$ aplicação realizada no AFWS, pode-se perceber uma característica já bastante conhecida dos modelos DEA tradicionais que é o seu fraco poder discriminatório quando se está trabalhando com um número reduzido de DMUs em relação ao número de critérios sendo considerados na análise, que no presente caso foram os modos de falha do sistema. Apesar disso, poder-se-ia ter aplicado algum modelo DEA alternativo como, por exemplo, o modelo de avaliação cruzada. No entanto, a aplicação do referido modelo não possibilitaria a discussão posterior, ou seja, de quanto cada índice precisa ser melhorado para que o

Tabela 8. Percentuais de melhoria para os índices dos modos de falha.

\begin{tabular}{cccc}
\hline Modos de Falha & Percentual O & Percentual G & Percentual D \\
\hline MF1 & 0 & 0 & 0 \\
MF2 & 0 & -40 & -50 \\
MF3 & 0 & -40 & -50 \\
MF4 & 0 & 0 & 0 \\
MF5 & 0 & 0 & 0 \\
MF6 & 0 & 0 & 0 \\
MF7 & -25 & -25 & 0 \\
MF8 & -25 & -25 & 0 \\
MF9 & 0 & 0 & 0 \\
MF10 & 0 & 0 & 0 \\
MF11 & -20 & -20 & -20 \\
MF12 & -20 & -20 & -20 \\
\hline
\end{tabular}

Tabela 9. Índices dos modos de falha (MF) do CVCS.

\begin{tabular}{cccccccccccc}
\hline MF & O & G & D & MF & O & G & D & MF & O & G & D \\
\hline $\mathbf{1}$ & 3 & 5 & 5 & $\mathbf{2 9}$ & 4 & 3 & 6 & $\mathbf{5 7}$ & 4 & 7 & 4 \\
$\mathbf{2}$ & 5 & 8 & 8 & $\mathbf{3 0}$ & 3 & 4 & 4 & $\mathbf{5 8}$ & 6 & 7 & 5 \\
$\mathbf{3}$ & 4 & 5 & 3 & $\mathbf{3 1}$ & 4 & 8 & 6 & $\mathbf{5 9}$ & 4 & 5 & 4 \\
$\mathbf{4}$ & 4 & 5 & 3 & $\mathbf{3 2}$ & 3 & 4 & 6 & $\mathbf{6 0}$ & 6 & 5 & 4 \\
$\mathbf{5}$ & 3 & 4 & 5 & $\mathbf{3 3}$ & 4 & 6 & 6 & $\mathbf{6 1}$ & 4 & 4 & 4 \\
$\mathbf{6}$ & 3 & 4 & 5 & $\mathbf{3 4}$ & 3 & 6 & 5 & $\mathbf{6 2}$ & 6 & 8 & 4 \\
$\mathbf{7}$ & 3 & 8 & 7 & $\mathbf{3 5}$ & 4 & 8 & 5 & $\mathbf{6 3}$ & 4 & 1 & 4 \\
$\mathbf{8}$ & 5 & 10 & 10 & $\mathbf{3 6}$ & 4 & 10 & 5 & $\mathbf{6 4}$ & 6 & 4 & 4 \\
$\mathbf{9}$ & 3 & 5 & 3 & $\mathbf{3 7}$ & 3 & 8 & 8 & $\mathbf{6 5}$ & 4 & 5 & 6 \\
$\mathbf{1 0}$ & 5 & 5 & 3 & $\mathbf{3 8}$ & 1 & 5 & 3 & $\mathbf{6 6}$ & 6 & 4 & 6 \\
$\mathbf{1 1}$ & 3 & 5 & 4 & $\mathbf{3 9}$ & 2 & 5 & 5 & $\mathbf{6 7}$ & 4 & 5 & 6 \\
$\mathbf{1 2}$ & 5 & 5 & 4 & $\mathbf{4 0}$ & 2 & 5 & 3 & $\mathbf{6 8}$ & 6 & 6 & 4 \\
$\mathbf{1 3}$ & 4 & 9 & 3 & $\mathbf{4 1}$ & 3 & 7 & 7 & $\mathbf{6 9}$ & 4 & 6 & 6 \\
$\mathbf{1 4}$ & 4 & 9 & 3 & $\mathbf{4 2}$ & 5 & 7 & 7 & $\mathbf{7 0}$ & 6 & 5 & 4 \\
$\mathbf{1 5}$ & 3 & 7 & 5 & $\mathbf{4 3}$ & 3 & 7 & 7 & $\mathbf{7 1}$ & 4 & 4 & 5 \\
$\mathbf{1 6}$ & 3 & 7 & 5 & $\mathbf{4 4}$ & 5 & 7 & 7 & $\mathbf{7 2}$ & 4 & 4 & 5 \\
$\mathbf{1 7}$ & 3 & 5 & 5 & $\mathbf{4 5}$ & 3 & 4 & 3 & $\mathbf{7 3}$ & 3 & 4 & 5 \\
$\mathbf{1 8}$ & 5 & 8 & 8 & $\mathbf{4 6}$ & 5 & 4 & 4 & $\mathbf{7 4}$ & 3 & 1 & 4 \\
$\mathbf{1 9}$ & 2 & 5 & 2 & $\mathbf{4 7}$ & 3 & 6 & 4 & $\mathbf{7 5}$ & 4 & 4 & 5 \\
$\mathbf{2 0}$ & 2 & 4 & 3 & $\mathbf{4 8}$ & 5 & 8 & 4 & $\mathbf{7 6}$ & 6 & 5 & 5 \\
$\mathbf{2 1}$ & 2 & 4 & 3 & $\mathbf{4 9}$ & 5 & 6 & 6 & $\mathbf{7 7}$ & 4 & 7 & 5 \\
$\mathbf{2 2}$ & 1 & 5 & 6 & $\mathbf{5 0}$ & 5 & 6 & 6 & $\mathbf{7 8}$ & 6 & 7 & 5 \\
$\mathbf{2 3}$ & 1 & 7 & 6 & $\mathbf{5 1}$ & 2 & 4 & 6 & $\mathbf{7 9}$ & 4 & 4 & 5 \\
$\mathbf{2 4}$ & 2 & 10 & 8 & $\mathbf{5 2}$ & 4 & 6 & 6 & $\mathbf{8 0}$ & 4 & 4 & 5 \\
$\mathbf{2 5}$ & 3 & 3 & 5 & $\mathbf{5 3}$ & 4 & 6 & 6 & $\mathbf{8 1}$ & 3 & 5 & 5 \\
$\mathbf{2 6}$ & 3 & 3 & 5 & $\mathbf{5 4}$ & 6 & 4 & 6 & $\mathbf{8 2}$ & 3 & 1 & 4 \\
$\mathbf{2 7}$ & 2 & 10 & 5 & $\mathbf{5 5}$ & 4 & 5 & 4 & - & - & - & - \\
$\mathbf{2 8}$ & 3 & 8 & 8 & $\mathbf{5 6}$ & 6 & 7 & 4 & - & - & - & - \\
\hline & & & & & & & & & & &
\end{tabular}


modo de falha deixe de ser considerado relativamente crítico. Esse fato se dá, pois, na avaliação cruzada, perde-se a fronteira, impossibilitando o cálculo dos percentuais de melhoria para cada um dos índices levantados durante a FMEA. Outra possibilidade, que se pretende investigar para aplicações futuras é a restrição aos pesos. Nesses modelos, pode-se trabalhar o poder discriminatório resguardando a possibilidade de se ter um direcionamento quanto aos percentuais de melhoria.

Quando o modelo DEA apresentado na formulação (1) foi aplicado a um sistema mais complexo, em que um número maior de modos de falha foi considerado, o poder discriminatório foi substancialmente melhorado.

Tabela 10. Índice de risco DEA e percentual de melhoria para os modos de falha (MF) do CVCS.

\begin{tabular}{|c|c|c|c|c|c|c|c|c|c|}
\hline MF & $\begin{array}{c}\text { Índice } \\
\text { DEA }\end{array}$ & $\% 0$ & $\% \mathrm{G}$ & $\% \mathrm{D}$ & MF & $\begin{array}{c}\text { Índice } \\
\text { DEA }\end{array}$ & $\% 0$ & $\% \mathrm{G}$ & $\% \mathrm{D}$ \\
\hline 1 & 65,96 & -34 & -34 & -34 & 42 & 44,93 & $-55,1$ & $-55,1$ & $-55,1$ \\
\hline 2 & 40,79 & $-59,2$ & $-59,2$ & $-59,2$ & 43 & 53,84 & $-46,2$ & $-46,2$ & $-52,7$ \\
\hline 3 & 81,78 & $-44,3$ & $-18,2$ & $-18,2$ & 44 & 44,93 & $-55,1$ & $-55,1$ & $-55,1$ \\
\hline 4 & 81,78 & $-44,3$ & $-18,2$ & $-18,2$ & 45 & 89,99 & $-21,7$ & -10 & -10 \\
\hline 5 & 70,45 & $-29,5$ & $-29,5$ & $-29,5$ & 46 & 74,96 & -50 & -25 & -25 \\
\hline 6 & 70,45 & $-29,5$ & $-29,5$ & $-29,5$ & 47 & 67,39 & $-32,6$ & $-32,6$ & $-32,6$ \\
\hline 7 & 50 & -50 & -50 & $-53,6$ & 48 & 56,23 & $-57,5$ & $-43,8$ & $-43,7$ \\
\hline 8 & 35 & -65 & -65 & $-66,2$ & 49 & 50 & -50 & -50 & -50 \\
\hline 9 & 81,81 & $-25,8$ & $-18,2$ & $-18,2$ & 50 & 50 & -50 & -50 & -50 \\
\hline 10 & 81,75 & $-55,5$ & $-18,2$ & $-18,2$ & 51 & 87,44 & $-12,5$ & $-12,5$ & $-43,7$ \\
\hline 11 & 72,09 & $-27,9$ & $-27,9$ & $-27,9$ & 52 & 53,45 & $-46,6$ & $-46,6$ & $-46,6$ \\
\hline 12 & 69,2 & $-52,3$ & $-30,8$ & $-30,8$ & 53 & 53,45 & $-46,6$ & $-46,6$ & $-46,6$ \\
\hline 13 & 66,61 & -50 & $-44,4$ & $-33,3$ & 54 & 56,23 & $-55,2$ & $-43,7$ & $-43,8$ \\
\hline 14 & 66,61 & -50 & $-44,4$ & $-33,3$ & 55 & 69,22 & $-40,4$ & $-30,8$ & $-30,8$ \\
\hline 15 & 58,49 & $-41,5$ & $-41,5$ & $-41,5$ & 56 & 59,96 & $-63,3$ & -40 & -40 \\
\hline 16 & 58,49 & $-41,5$ & $-41,5$ & $-41,5$ & 57 & 59,99 & -45 & -40 & -40 \\
\hline 17 & 65,96 & -34 & -34 & -34 & 58 & 52,92 & $-61,3$ & $-47,1$ & $-47,1$ \\
\hline 18 & 40,79 & $-59,2$ & $-59,2$ & $-59,2$ & 59 & 69,22 & $-40,4$ & $-30,8$ & $-30,8$ \\
\hline 19 & 100 & 0 & 0 & 0 & 60 & 69,17 & $-60,3$ & $-30,8$ & $-30,8$ \\
\hline 20 & 96,88 & $-3,1$ & $-3,1$ & $-3,1$ & 61 & 74,98 & $-37,5$ & -25 & -25 \\
\hline 21 & 96,88 & $-3,1$ & $-3,1$ & $-3,1$ & 62 & 56,22 & $-64,6$ & $-43,8$ & $-43,7$ \\
\hline 22 & 99,89 & 0,1 & 0 & -50 & 63 & 99,95 & -25 & 0,1 & 0 \\
\hline 23 & 99,64 & 0 & $-28,6$ & -50 & 64 & 74,93 & $-58,3$ & -25 & -25 \\
\hline 24 & 49,98 & -50 & -50 & $-62,5$ & 65 & 56,36 & $-43,6$ & $-43,6$ & $-43,6$ \\
\hline 25 & 77,77 & $-22,2$ & $-22,2$ & $-26,7$ & 66 & 56,23 & $-55,2$ & $-43,7$ & $-43,8$ \\
\hline 26 & 77,77 & $-22,2$ & $-22,2$ & $-26,7$ & 67 & 56,36 & $-43,6$ & $-43,6$ & $-43,6$ \\
\hline 27 & 57,13 & $-42,8$ & -50 & $-42,9$ & 68 & 64,24 & $-61,9$ & $-35,7$ & $-35,7$ \\
\hline 28 & 49,99 & -50 & -50 & $-59,4$ & 69 & 53,45 & $-46,6$ & $-46,6$ & $-46,6$ \\
\hline 29 & 63,64 & $-36,4$ & $-36,4$ & $-37,1$ & 70 & 69,17 & $-60,3$ & $-30,8$ & $-30,8$ \\
\hline 30 & 77,5 & $-22,5$ & $-22,5$ & $-22,5$ & 71 & 64,58 & $-35,4$ & $-35,4$ & $-35,4$ \\
\hline 31 & 48,44 & $-51,6$ & $-51,6$ & $-51,6$ & 72 & 64,58 & $-35,4$ & $-35,4$ & $-35,4$ \\
\hline 32 & 69,98 & -30 & -30 & $-40,8$ & 73 & 70,45 & $-29,5$ & $-29,5$ & $-29,5$ \\
\hline 33 & 53,45 & $-46,6$ & $-46,6$ & $-46,6$ & 74 & 100 & 0 & 0 & 0 \\
\hline 34 & 62 & -38 & -38 & -38 & 75 & 64,58 & $-35,4$ & $-35,4$ & $-35,4$ \\
\hline 35 & 51,67 & $-48,3$ & $-48,3$ & $-48,3$ & 76 & 59,97 & $-58,3$ & -40 & -40 \\
\hline 36 & 46,97 & -53 & -53 & -53 & 77 & 54,39 & $-45,6$ & $-45,6$ & $-45,6$ \\
\hline 37 & 49,99 & -50 & -50 & $-59,4$ & 78 & 52,92 & $-61,3$ & $-47,1$ & $-47,1$ \\
\hline 38 & 100 & 0 & 0 & 0 & 79 & 64,58 & $-35,4$ & $-35,4$ & $-35,4$ \\
\hline 39 & 77,76 & $-22,2$ & $-22,2$ & $-34,4$ & 80 & 64,58 & $-35,4$ & $-35,4$ & $-35,4$ \\
\hline 40 & 88,57 & $-11,4$ & $-11,4$ & $-11,4$ & 81 & 65,96 & -34 & -34 & -34 \\
\hline 41 & 53,84 & $-46,2$ & $-46,2$ & $-52,7$ & 82 & 100 & 0 & 0 & 0 \\
\hline
\end{tabular}


Entre os 82 modos de falha identificados para o CVCS, somente 4 foram considerados relativamente não críticos.

Apesar da possibilidade de se identificar o quanto cada índice deve ser reduzido para que o modo de falha se torne relativamente não crítico, uma discussão que precisa ser feita está relacionada com as atividades e investimentos no sistema que precisam se dar para que esses índices sejam efetivamente reduzidos. Por exemplo, o que significa ter que realizar uma redução de 50\% num índice de gravidade? Lembrando que o índice de gravidade está relacionado com as consequências da ocorrência do modo de falha, uma redução nesse índice está relacionada com uma redução nos efeitos. Para se alcançar essas medidas de redução, uma análise das camadas de proteção (LOPA - Leyer of Protection Analysis) pode ser de grande valia.

As mesmas discussões devem ser feitas para os demais índices. Por exemplo, o que significa uma redução de $\mathrm{X} \%$ no índice de ocorrência? A redução na frequência de ocorrência de um determinado modo de falha pode estar relacionada com o crescimento necessário para a confiabilidade do componente ou do subsistema sob análise. Para tanto, uma análise custo vs benefício deve ser realizada, identificando o quanto se ganha com o crescimento da confiabilidade e qual o custo para se alcançar esse crescimento. De maneira similar, deve-se tentar responder o que significa uma redução de Y\% no índice de detecção. O índice de detecção está relacionado com os controles do sistema. Uma análise dos níveis de integridade de segurança (SIL - Safety Integrity Level) pode auxiliar sobremaneira na identificação dos potenciais de melhoria nos controles de segurança do sistema.

As discussões suscitadas por meio da análise dos modos de falha por meio da DEA podem auxiliar de forma eficiente nas análises de segurança de instalações industriais. $\mathrm{O}$ presente artigo demonstrou, por meio dos exemplos práticos, a viabilidade de se utilizar os modelos DEA convencionais para auxiliar na identificação de pontos críticos, com base numa FMEA, além de permitir identificar o quanto e de que forma cada um desses pontos deve ser melhorado. Obviamente que se trata de uma abordagem de apoio à decisão e que análise complementares conforme as supracitadas são de suma importância para a qualidade dos resultados efetivos sobre o sistema sob análise.

\section{Agradecimentos}

$\mathrm{O}$ autor do trabalho gostaria de agradecer à FAPERJ por ter apoiado o desenvolvimento desta pesquisa por meio do projeto E-26/110-327/2011 de Auxílio à Pesquisa Básica.

\section{Referências}

BEVILACQUA, M.; BRAGLIA, M.; GABBRIELLI, R. Monte Carlo simulation approach for a madified FMECA in Power plant. Quality and Reliability Engineering International, v. 16, p. 313-324, 2000. http://dx.doi. org/10.1002/1099-1638(200007/08)16:4\%3C313::AIDQRE434\%3E3.0.CO;2-U

BOWLES, J. B. The new SAE FMECA standard. In: IEEE ANNUAL RELIABILITY AND MAINTAINABILITY SYMPOSIUM, 1998, Anaheim. Proceedings... Anaheim: IEEE, 1998. p. 48-53.

BOWLES, J. B. An assessment of RPN prioritization in failure modes, effect and criticality analysis. In: IEEE ANNUAL RELIABILITY AND MAINTAINABILITY SYMPOSIUM, 2003, Tampa. Proceedings... Tampa: IEEE, 2003. http://dx.doi.org/10.1109/ RAMS.2003.1182019

BOWLES, J. B.; BONNELL, R. D. Failure mode, effect and criticality analysis: what it is and how to use it. In: TOPIC IN RELIABILITY AND MAINTAINABILITY AND STATISTICS, ANNUAL RELIABILITY AND MAINTAINABILITY SYMPOSIUM, 1998, Anaheim. Proceedings... Anaheim: IEEE, 1998.

BOWLES, J. B.; PELÁEZ, C. E. Fuzzy logic prioritization in a system failure mode, effect and criticality analysis. Reliability Engineering and System Safety, v. 50, n. 2, p. 203-213, 1995. http://dx.doi. org/10.1016/0951-8320(95)00068-D

BOWLES, J. B.; PELÁEZ, C. E. Using fuzzy cognitive maps as a system model for failure modes and effect analysis. Information Science, v. 88, n. 1-4, p. 177-199, 1996. http://dx.doi.org/10.1016/0020-0255(95)00161-1

BRAGLIA, M.; FROSOLINI, M.; MONTANARY, R. Fuzzy criticality assessment model for failure modes and effect analysis. International Journal of Quality and Reliability Management, v. 20, n. 4, p. 503-524, 2003. http://dx.doi.org/10.1108/02656710310468687

COOPER, W. W.; SEIFORD, L. M.; TONE, K. Data Envelopment Analysis: A comprehensive text with models, applications, references and DEA-solver software. 2nd ed. New York: Springer, 2007.

CHANG, C. L.; LIU, P. H.; WEI, C. C. Failure mode and effect analysis using grey theory. Integrated Manufacturing Systems, v. 12, n. 3, p. 211-216, 2001. http://dx.doi.org/10.1108/09576060110391174

CHARNES, A.; COOPER, W. W.; RHODES, E. Measuring The Efficiency of Decision Making Units. European Journal of Operational Research, n. 2, p. 429-444, 1978. http://dx.doi.org/10.1016/0377-2217(78)90138-8

CHARNES, A. et al. Data Envelopment Analysis: Theory, methodology and applications. Norwell: Kluwer Academic Publishers, 1994.

CHENG, C. L.; WEI, C. C.; LEE, Y. H. Failure mode and effect analysis using fuzzy method and grey theory. Kybernetes, v. 28, p. 1072-1080, 1999. http://dx.doi. org/10.1108/03684929910300295

CHIN, K. S. et al. Failure mode and effects analysis using a group-based evidential reasoning approach. Computers and Operations Research, v. 36, n. 6, p. 1768-1779, 2009a. http://dx.doi.org/10.1016/j.cor.2008.05.002 
CHIN, K. S. et al. Failure mode and effects analysis by data envelopment analysis. Decision Support Systems, v. 48, n. 1, p. 246-256, 2009b. http://dx.doi.org/10.1016/j. dss.2009.08.005

ELECTRIC POWER RESEARCH INSTITUR - EPRI. TR-106706 Risk informed inservice inspection evaluation procedure. EPRI, 1996.

FARRELL, M. J. The Measurement of Productive Efficiency. Journal of the Royal Statistical Society, Series A, part III, p. 253-290, 1957. http://dx.doi.org/10.2307/2343100

FULLWOOD, R. R. Probabilistic risk assessment in chemical and nuclear industries. Woburn: ButterworthHeinemann, 2000. PMCid:1186920.

GARCIA, P. A. A. Aplicação de Análise Envoltória de Dados - (DEA) no Processo de Manutenção Centrada em Confiabilidade - (RCM). 2001. 78 f. Dissertação (Mestrado em Sistema e Computação)-Instituto Militar de Engenharia, Rio de Janeiro, 2001.

GARCIA, P. A. A.; NEVES, J. C. S. DEA: a novel approach to failure mode evaluation in RCM. In: SIMPÓSIO DE PESQUISA OPERACIONAL E LOGÍSTICA DA MARINHA, 2001, Rio de Janeiro. Anais... Rio de Janeiro, 2001.

GARCIA, P. A. A.; NEVES, J. C. S.; LUZ, V. Aplicação de um Modelo DEA para Priorizar Modos de Falha. In: ENCONTRO NACIONAL DE ENGENHARIA DE PRODUÇÃO, 21., 2001, Salvador. Anais... Salvador, 2001. p. 188. PMid:11152800.

GARCIA, P. A. A.; SCHIRRU, R.; FRUTUOSO E MELO, P. F. F. A fuzzy data envelopment analysis approach for FMEA. Progress in Nuclear Energy, v. 46, n. 3-4, p. 359-373, 2005. http://dx.doi.org/10.1016/j. pnucene.2005.03.016

GARCIA, P. A. A.; FRUTUOSO E MELO, P. F. F.; SCHIRRU, R. Aplicação de um modelo fuzzy DEA para priorizar modos de falha em sistemas nucleares. Pesquisa Operacional, v. 29, n. 2, p. 382-402, 2009.

GARCIA, P. A. A.; LEAL JUNIOR, I. C.; OLIVEIRA, M. A. A Linear Programming Approach to Risk Prioritization in FMEA. In: EUROPEAN SAFETY AND RELIABILITY CONFERENCE - ESREL, 2012, Troyes. Proceedings... London: Taylor \& Francis Group, 2012. p. 1541-1546.

GOMES JÚNIOR, S. F.; SOARES DE MELLO, J. C. C. B. Utilização de modelo DEA com restrições cone ratio não arquimedianas para avaliação dos pilotos no campeonato mundial de fórmula 1 do ano de 2006. Sistema \& Gestão, v. 2 n. 3, p. 217-231, 2007.

GUIMARÃES, A. C. F.; LAPA, C. M. F. Effect analysis fuzzy inference system in nuclear problems using approximate reasoning. Annals of Nuclear Energy, v. 31, p. 107-115, 2004a. http://dx.doi.org/10.1016/ S0306-4549(03)00173-7
GUIMARÃES, A. C. F.; LAPA, C. M. F. Fuzzy FMEA applied to PWR chemical and volumetric control system. Progress in Nuclear Energy, v. 44, n. 31, p. 191-213, 2004b. http://dx.doi.org/10.1016/S0149-1970(04)90010-8

GUIMARÃES, A. C. F.; LAPA, C. M. F. Fuzzy inference to risk assessment on nuclear engineering systems. Applied Soft Computing, v. 7, p. 17-28, 2007. http:// dx.doi.org/10.1016/j.asoc.2005.06.002

INSTITUTO DE QUALIDADE AUTOMOTIVA - IQA. QS-9000: Análise dos modos e efeitos de falha em processo e Análise dos modos e efeitos de falha em produtos. IQA, 1997. Manual de Referência.

INTERNATIONAL ATOMIC ENERGY AGENCY - IAEA. Procedure for conducting probabilistic safety assessments of nuclear power plants (level 1): a safety practice. Vienna: IAEA, 1992. p. 4. (Safety Series, n. 50).

INTERNATIONAL ATOMIC ENERGY AGENCY - IAEA. Applications of probabilistic safety assessment (PSA) for nuclear power plant. Vienna: IAEA, 2001. Technical Document 1200.

INTERNATIONAL ATOMIC ENERGY AGENCY - IAEA. Procedure for conducting probabilistic safety assessment for non-reactor nuclear facilities. Vienna: IAEA, 2002. Technical Document 1267.

LINS, M. P. E.; ANGULO MEZA, L. A. Análise envoltória de dados e perspectivas de integração no ambiente de apoio à decisão. Rio de Janeiro: COPPE/UFRJ, 2001.

LOVELL, C. A. K.; PASTOR, J. T. Radial DEA Models Without Inputs or Without Outputs. European Journal of Operational Research, n. 118, p. 46-51, 1999. http:// dx.doi.org/10.1016/S0377-2217(98)00338-5

SANKAR, N. R.; PRABHUR, B. S. Modified approach for prioritization of failures in a system failure mode and effect analysis. International Journal of Quality and Reliability Managemant, v. 18, n. 3, p. 324-335, 2001. http://dx.doi.org/10.1108/02656710110383737

SOARES DE MELLO, J. C. C. B. Modelos DEA de input unitário com aparente orientação a inputs. In: SIMPÓSIO BRASILEIRO DE PESQUISA OPERACIONAL, 38., 2006, Goiânia. Anais... Goiânia: Sociedade Brasileira de Pesquisa Operacional, 2006.

SOCIETY FOR AUTOMOTIVE ENGINEERS - SAE. J-1739: Potential Failure Mode and Effects Analysis in Design, Potential Failure Mode and Effects Analysis in Manufacturing and Assembly Processes. SAE, 2009.

YANG, Z.; BONSALL, S.; WANG, J. Fuzzy rule-based Bayesian reasoning approach for prioritization of failures in FMEA. IEEE Transaction on Reliability, v. 57, n. 3, p. 517-528, 2008. http://dx.doi.org/10.1109/ TR.2008.928208 